\title{
Long range annealing of defects in germanium by low energy plasma ions
}

\author{
J. F. R. Archilla ${ }^{\mathrm{a}, *}$, S. M. M. Coelho ${ }^{\mathrm{b}}$, F. D. Auret $^{\mathrm{b}}$, V. I. Dubinko ${ }^{\mathrm{c}}$, V. Hizhnyakov ${ }^{\mathrm{d}}$ \\ ${ }^{a}$ Group of Nonlinear Physics, Universidad de Sevilla, ETSII, Avda Reina Mercedes s/n, \\ 41012-Sevilla, Spain \\ ${ }^{b}$ Department of Physics, University of Pretoria, Lynnwood Road, Pretoria 0002, South Africa \\ ${ }^{c}$ NSC Kharkov Institute of Physics and Technology, Kharkov 61108, Ukraine \\ ${ }^{d}$ Institute of Physics, University of Tartu, Riia 142, EE-51014 Tartu, Estonia
}

\begin{abstract}
Ions arriving at a semiconductor surface with very low energy $(2-8 \mathrm{eV})$ are interacting with defects deep inside the semiconductor. Several different defects were removed or modified in Sb-doped germanium, of which the $E$-center has the highest concentration. The low fluence and low energy of the plasma ions implies that the energy has to be able to travel in a localized way to be able to interact with defects up to a few microns below the semiconductor surface. After eliminating other possibilities (electric field, light, heat) we now conclude that moving intrinsic localized modes (ILMs), as a mechanism of longdistance energy transport, are the most likely cause. This would be striking evidence of the importance of ILMs in crystals and opens the way to further experiments to probe ILM properties both in semiconductors and in the metals used for contacts. Although most of the measurements have been performed on germanium, similar effects have been found in silicon.
\end{abstract}

Keywords: Germanium, ILM, discrete breathers, quodons, defects, DLTS

PACS: 63.20.Pw, 63.20.Ry, 63.50.+x, 66.90.+r, 82.20.-w

\section{Introduction}

There is increasing interest in the mechanism and properties of non-linear lattice vibrations in crystals, known as intrinsic localized modes (ILMs) [1], discrete breathers (DBs) [2] and quodons [3]. They have been proposed as a mechanism to facilitate various reactions deep in solids $[4,5]$.

They can be stationary and may have long lifetimes and large propagation distances [3]. In recent works, existence of stationary [6] as well as mobile ILMs [7] has been demonstrated in metals by means of molecular dynamics (MD). A key point has been the introduction of modern potentials obtained with the embedded-atom method

* Corresponding author

Email address: archilla@us.es (J. F. R. Archilla) 
(EAM) [8] that takes into account the electron charge density. EAM potentials often show a local hardening of the atom pair potential which makes possible the existence of ILMs above the phonon spectrum. ILMs obtained with MD have energies ranging from fractions of an $\mathrm{eV}$ to a few $\mathrm{eV}$; and therefore may be readily produced in low-energy atomic collisions that do not result in permanent atomic displacements.

In order to observe them experimentally it is necessary to have a) a source of localized energy of the order of an eV, b) a localized property which may be affected by energies of that order and c) a technique to observe those changes. Localized energies of the order of an eV can be transmitted by low density plasmas with low energy ions. Some defects in solids have annealing energies of order of $\mathrm{eV}$ and there exist techniques to measure the concentration of some of them inside a solid.

Deep Level Transient Spectroscopy (DLTS) $[9,10]$ is an especially useful technique for direct experimental observation of such phenomena in semiconductors, since it allows one to detect microstructural changes deep below inside the material produced by low-energy collision events at the surface. Using this technique, some of the authors have found that low energy plasma acting on germanium produces the annealing of defects deep below the metal-semiconductor interface, showing that energy is transmitted distances of microns in a localized way.

In this paper we describe this experiment with some detail, examining possible explanations and suggest that the evidence is compelling that ILMs are the cause of these observations.

\subsection{Defect detection by DLTS}

DLTS detects defects that are electrically active, namely that act as electron or hole donors, or traps in the usual terminology. A defect lying in the band gap with energy $>0.1 \mathrm{eV}$ from either band edge is termed deep. See Refs. [11, 12, 13, 14] for properties of defects in Ge. For simplicity, we will refer to electron traps, although a similar description is valid for hole traps. When an electron is in the trap level it has some probability of being emitted to the conduction band. The emission rate is given by $[9,10]$ :

$$
e_{n}=\sigma_{\mathrm{app}} N_{c} v_{\mathrm{th}} \exp \left(-E_{T} / k_{B} T\right)
$$

where $\sigma_{\text {app }}$ is the apparent capture cross section, $N_{c}$ is the effective density of states in the conduction band, $v_{\mathrm{th}}$ is the mean velocity of the electrons and $E_{T}$ is the difference in energy from the bottom of the conduction band to the trap level inside the band gap. $E_{T}$ is the activation energy for the emission process and it should not be confused with the energy for creating or annealing the defect. The lifetime, $\tau$, of an electron in the trap is the inverse of the emission rate, $\tau=1 / e_{n}$. In this paper a prominent defect will be the $E$-center which consists of an association of a vacancy and a substitutional donor, Sb in our experiments. The em E-center has three levels of which one dominates our DLTS spectra, corresponding to an electron trap with $E_{T}=0.37 \mathrm{eV}$.

Deposition of a thin layer of metal on the semiconductor surface produces a Schottky diode or an ohmic contact, a rectifying junction being a prerequisite to perform measurements provided that both contacts are present.

Following an injection pulse of carriers the traps are rapidly filled and subsequently release carriers. The capacitance at two different times $t_{1}$ and $t_{2}$ is measured, the change in capacitance $\Delta C=C\left(t_{2}\right)-C\left(t_{1}\right)$ has a maximum when the emission rate of a defect 


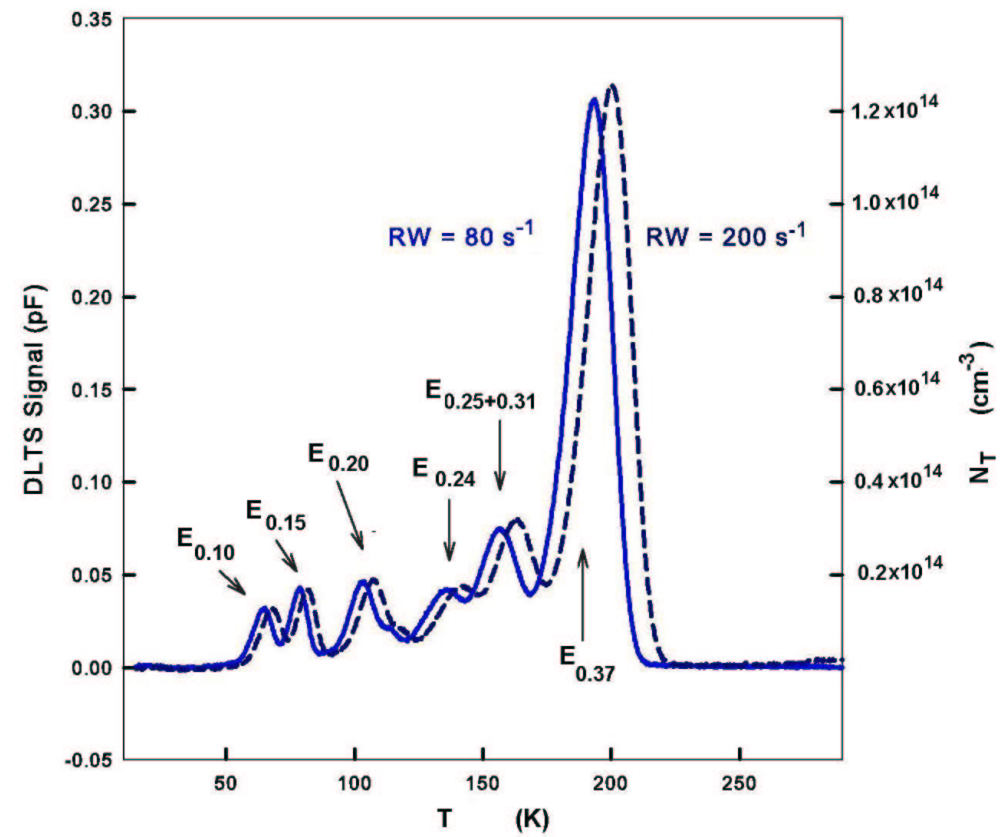

Figure 1: (Color online) Spectra obtained in alpha particle damaged Ge for two rate windows $80 \mathrm{~s}^{-1}$ and $200 \mathrm{~s}^{-1}$ and the defects observed. The labels at the right Y-axis indicate the defect concentration at the respective peaks and are meaningless otherwise.

equals the so-called rate window $R W=\ln \left(t_{2} / t_{1}\right) /\left(t_{2}-t_{1}\right)$. Rate windows are preset at definite values, typically 80 or $200 \mathrm{~s}^{-1}$. As the emission rate $e_{n}$ of a defect depends strongly on the temperature $T$, by changing it, it is possible to make $e_{n}$ equal to the preset rate window, which appears as a maximum of $\Delta C$ as a function of $T$, for a given temperature. Different defects will bring about maxima at different temperatures. Setting two or more different RWs will lead to two different peak temperatures for a given defect and allows one through an Arrhenius plot of $\ln \left(e_{n} / T^{2}\right)$ versus $1 / T$ to determine $E_{T}$. Likewise $\sigma_{\text {app }}$ can be obtained and the defect can be fully characterized [14]. For clarity, only the RW $80 \mathrm{~s}^{-1}$ will be presented in this article unless stated otherwise.

The height of the DLTS peak is proportional to the defect concentration as $N_{T}=$ $2 N_{D}(\Delta C / C)_{\text {Peak }}, N_{D}$ being the concentration of donor atoms. In Figure 1 the DTLS signal for two rate windows $80 \mathrm{~s}^{-1}$ and $200 \mathrm{~s}^{-1}$ and the trap energies of the defects in the damaged Ge wafer can be seen, as will be explained below.

An important property of the technique is that the reverse bias of the diode determines the size of the depletion layer and allows for the detection of how deep inside the semiconductor the defects are and even to obtain the dependence of the concentration with depth. 


\subsection{Rate of ion-induced annealing}

We can describe the rate of annealing by ion bombardment by the following equation:

$$
\frac{\mathrm{d} N_{T}}{\mathrm{~d} t}=-\sigma_{\mathrm{i}} N_{T} \Phi_{\mathrm{i}}
$$

where $N_{T}$ is the number of defects or traps of a specific type, which in this paper will be mainly the $E$-center and $\Phi_{\mathrm{i}}$ is the flux of ions on the surface of the semiconductor. The magnitude $\sigma_{\mathrm{i}}$ is an effective ion-defect cross-section, as if imaginary ions entered the crystal and interacted with the defects. Therefore $\sigma_{\mathrm{i}}$ measures the efficiency of the ions in annealing defects, whatever the mechanism. The flux of plasma ions can be measured directly with a Faraday cup. The number of defects $N_{T}$ is measured with DLTS before and after time $t$ of ICP, typically 10 or 30 minutes, the latter being more precise but care has to be taken to prevent heating, thus the choice to anneal in 10 minute intervals is justified or essential. Therefore:

$$
N_{T}(t)=N_{T}(0) \exp \left(-\sigma_{\mathrm{i}} \Phi_{\mathrm{i}} t\right)
$$

from which $\sigma_{\mathrm{i}}$ and the instantaneous annealing rate can be easily obtained.

\section{Experimental procedure}

The semiconductor used is Ge doped with $\mathrm{Sb}$ with a concentration $n_{\mathrm{Sb}}=1.3 \times$ $10^{15} \mathrm{~cm}^{-3}$. The typical size of the Ge wafer is $3 \times 5 \times 0.6 \mathrm{in} \mathrm{mm}$.

Defects are created by damaging the semiconductor with $5 \mathrm{MeV} \alpha$ particles (Americium foil). Subsequently a thin layer of metal, with typical thickness of about $25 \mathrm{~nm}$, is deposited using resistive evaporation ( $\mathrm{RE}$ ), a technique which is known not to introduce defects in semiconductors. Up to a depth exceeding $2600 \mathrm{~nm}$, the defect concentration in the sample diminishes slightly with depth as seen in Figure 5. AuSb was evaporated previously onto the back surface to form the Ohmic contact.

The observed process of non-thermal annealing is obtained when the semiconductor is treated with an inductively coupled plasma (ICP) of Ar. As can be seen in Fig. 4 several defects are annealed or transformed. Here we will focus on the most abundant defect corresponding to the highest peak at $185 \mathrm{~K}$. The first few hours there is a very fast kinetics which is explained by a mobile species that consumes $E$-centers and which is produced by an unstable source created during alpha particle irradiation [14].

After 6 hours at room temperature a slower kinetics is established as can be seen in Figure 3. Our experiments are produced after the first kinetics has stopped.

To summarize the experimental procedure: 1) Damage material with $5 \mathrm{MeV}$ alpha particles. 2) Rest 24 hours at room temperature to anneal out the unknown defect. 3) Evaporate $\mathrm{Au}$ diodes $(25 \mathrm{~nm}$ ) on half the sample (diode A) and measure defects. 4) ICP-treat sample (4 eV Ar ions) for 30 minutes at room temperature in intervals of 10 minutes to prevent heating. 5) Evaporate Au diodes on the second half of the sample (diode B). 6) Perform DLTS on diode A. 7) DLTS on diode B.

Therefore, three DLTS spectra were obtained, as seen in Fig. 4, with the following results: 


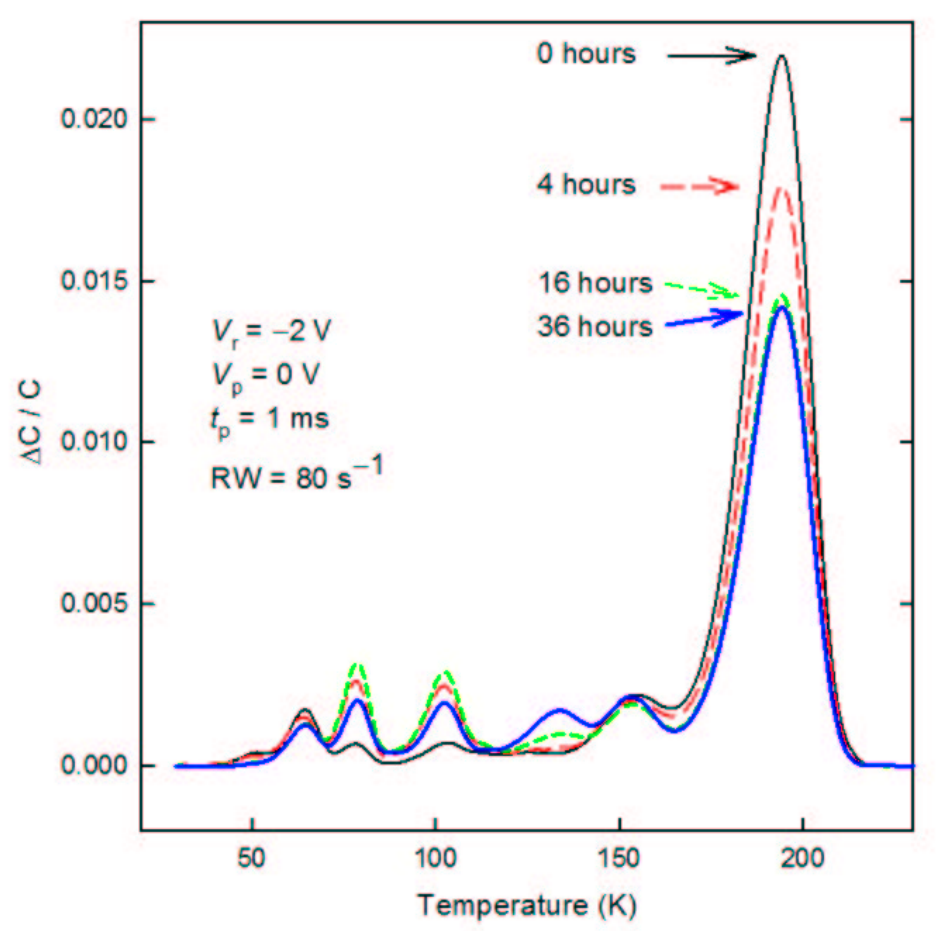

Figure 2: (Color online) Five DLTS spectra performed in Sb-doped Ge after being damaged by $5 \mathrm{MeV}$ $\alpha$ particles followed by 24 hours of being annealed at room temperature. It can be observed that the temperature height of the highest peak decreases markedly in the first 16 hours.

1) E-center concentration, initially at $N_{T}=1.07 \times 10^{14} \mathrm{~cm}^{-3}$, fell approximately $30 \%$ after ICP on germanium. When the ICP was done through the Au contact, the reduction in concentration was about $7 \%$ but still evident.

2) Resistive evaporation of metals onto Ge also reduced the defect concentration. These changes in defect concentration were metal dependent.

3) The sample heats up a bit to about $40^{\circ} \mathrm{C}$, although the ICP is done in 10 minute steps to allow cooling. If the ICP treatment is done continuously the sample heats up more $\left(\simeq 65^{\circ} \mathrm{C}\right)$ and the effect of ICP annealing diminishes dramatically showing that heating is not the cause.

4) The defects were annealed up to a depth exceeding $2600 \mathrm{~nm}$ below the Ge surface. With $5.66 \AA$ lattice constant, it means that the effect of the Ar plasma is felt more than 4500 lattice units deep. Figure 5 shows the concentration profile as a function of depth for the $E$-center before and after ICP.

5) The rate of annealing increases with the energy of the plasma, because the flux also increases, but the ion-defect cross section $\sigma_{\mathrm{i}}$ defined in Eq. 2 decreases strongly with the ion energy, being 130 times smaller for $8 \mathrm{eV}$ than for $4 \mathrm{eV}$ ions. Apparently it is also larger for $2 \mathrm{eV}$ ions, although the measurements are not precise as the ion flux and 


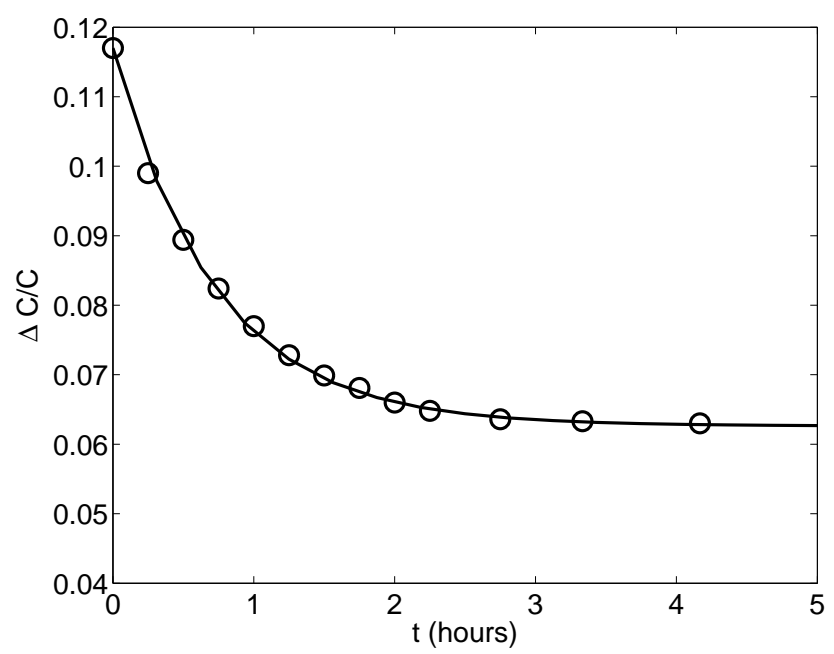

Figure 3: DLTS signal of $E_{0.38}$ (E-center) during the first few hours of room temperature annealing. It can be seen that the defect has very fast kinetics initially as explained in Ref. [14]. See text.

annealing rate are very small.

5) The temperature to achieve similar rate of annealing is about $150^{\circ} \mathrm{C}[13,15]$.

\subsection{Possible explanations}

In this section we analyze possible explanations to our experiment and comment why they can be discarded.

1) Dislocations could be introduced by alpha damage but not by the plasma treatment being used as the energy was too low [16]. Also the alpha particle dose was too low to produce multivacancies $[17,18]$

2) Low energy argon impacts may produce minority charge carriers which are known to anneal out defects near the surface. However, if this was the case, the effect should be limited to the neighborhood of the surface, while we have an almost constant annealing profile. Also, although we report mainly on the $E$-center, we observe changes to all of the point defects that are present, whereas electronically stimulated defect migration has been reported only for specific defects [19]

3) The reduction in the defect concentration is almost uniform with depth up to $2.6 \mathrm{~nm}$, meaning that the annealing is not a surface or subsurface process and is almost proportional to the local defect concentration as demonstrated in Figure 6.

4) It can be argued that low energy ion bombardment can induce defects which may diffuse into the bulk and form electrically active complexes. However, it has been shown that it only produces one defect, $\mathrm{E}_{0.31}[16]$ and this defect does not diffuse into the material. The defect introduced during ICP is a subthreshold defect, created when a pre-existing defect site is modified, that only diffuses into Ge at a temperature or length of time far exceeding that experienced during ICP. Here we report on the plasma interacting with different defect sites. 


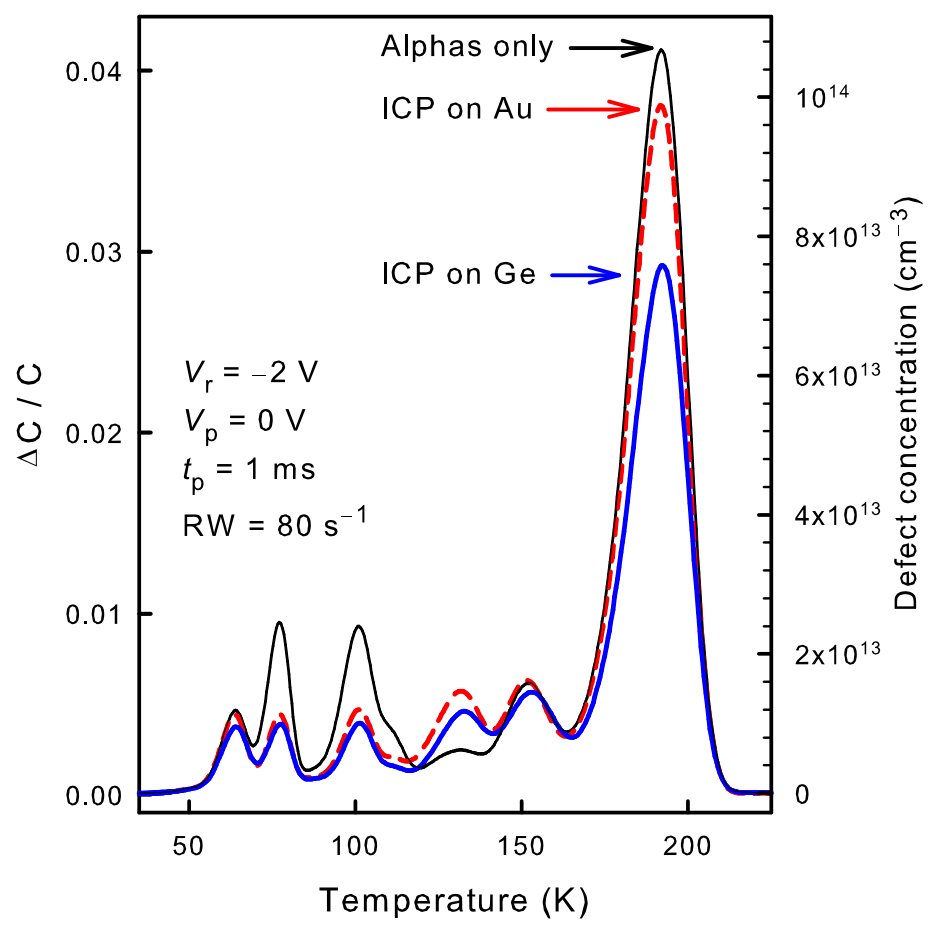

Figure 4: (Color online) Three DLTS spectra performed in Sb-doped Ge after being damaged by $5 \mathrm{MeV}$ $\alpha$ particles followed by 24 hours of room temperature annealing. The labels at the right Y-axis indicate the defect concentration at the respective peaks and are meaningless otherwise. Black (thin line): before ICP; red (dashed): after 30' ICP through an Au contact; blue (thick line): after 30' ICP directly on Ge. See text for explanation. The highest peak at $185 \mathrm{~K}$ corresponds to the $E$-center defect.

5) Another possibility that might be thought of is that hydrogen contained in a layer of native oxide could be released during the low energy ion bombardment and diffuses deep into the substrate where they could partially passivate existing vacancy type defects. But hydrogen passivation of defects in Ge has been shown to be different to that in Si. Hydrogen is unable to passivate dangling bonds in Ge, since hydrogen, is exclusively negatively charged [20, 21].

6) Channelling of Ar in Ge has been found in molecular dynamics by some of the authors. However, using Auger electron spectroscopy it was not possible to find any trace of Ar after ICP and it has been shown that similar long range energy transmission occurs with electron beam deposition and exposure where Ar is not involved [22]. Also, the effect was independent of crystal orientation [16] while channelling should depend on it.

\subsection{Moving ILM hypothesis}

In the following we explore the possibility that moving intrinsic localized modes could be the long range carriers of localized energy. That is, that Ar ions or neutrals impacting 


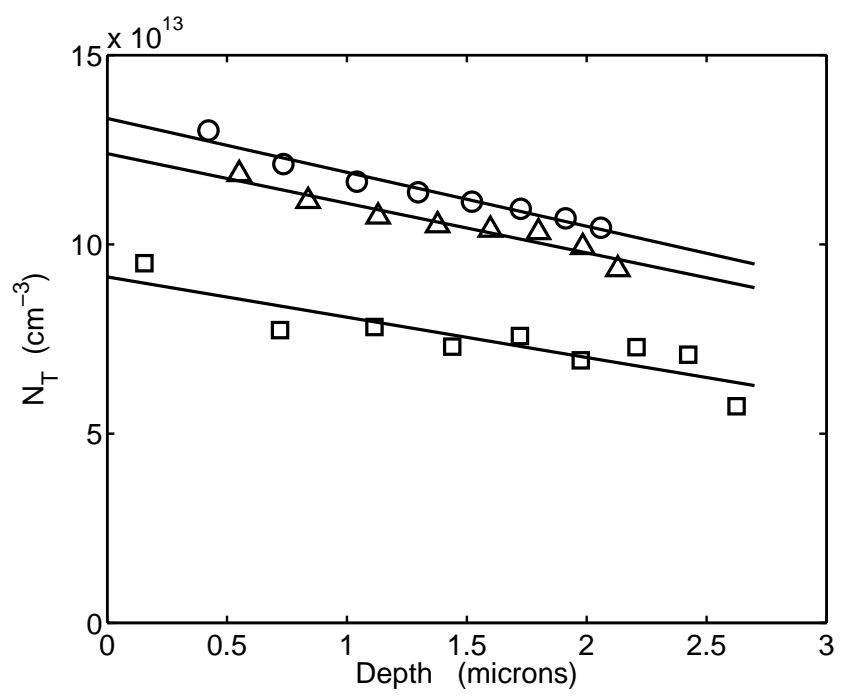

Figure 5: Profile of the concentration of the $E$-center defect after alpha particle damage (circles), after 30 minutes ICP on Au (triangles) and after the same time of ICP directly on Ge (squares). Lines are linear fits.

on the Ge surface are able to produce moving ILMs and the latter can interact with defects, either annealing of modifying them. The reasons for this hypothesis are the following:

1) ILMs obtained in solids with molecular dynamics have energies of about $0.5-5 \mathrm{eV}[6,7]$ depending on the material. The maximum energy transfer from a $4 \mathrm{eV} \mathrm{Ar}$ ion to a $\mathrm{Ge}$ atom is about $3.7 \mathrm{eV}$.

2) The perturbation has to be localized, because if not it will spread into the semiconductor transforming into phonons, with much less annealing capability as explained below.

3 ) The energy for annealing a defect is of the order of an $\mathrm{eV}$ and can be obtained from annealing curves in Ref. [13], and density functional theory [23]. A specific value of $1.36 \mathrm{eV}$ is given in Ref. [15]. Therefore, a large part of the ILM energy has to survive several thousand lattice units. This is impossible with wave packets and phonons, while large amplitude ILMs have been shown to decay slowly through a two-phonon process [24].

4) Increasing the energy of the Ar plasma to $8 \mathrm{eV}$ does result in doubling the rate of annealing, however as the flux of ions from the ICP plasma increases by more than $240 \%$, the annealing effect is actually two orders of magnitude lower. This is typical of ILMs, which usually have a limited range of amplitudes and energies for their existence. Ions of larger energy may be worse at producing ILMs if the latter have significantly smaller energy, because the energy not used will create phonons and a local increase of temperature which destroys the ILMs.

5) At least stationary ILMs have been constructed for Si and Ge [25] using molecular dynamics.

These hypotheses also have implications for the metals used as contacts, as they are also able to transmit localized perturbations to the semiconductor with an efficiency of 


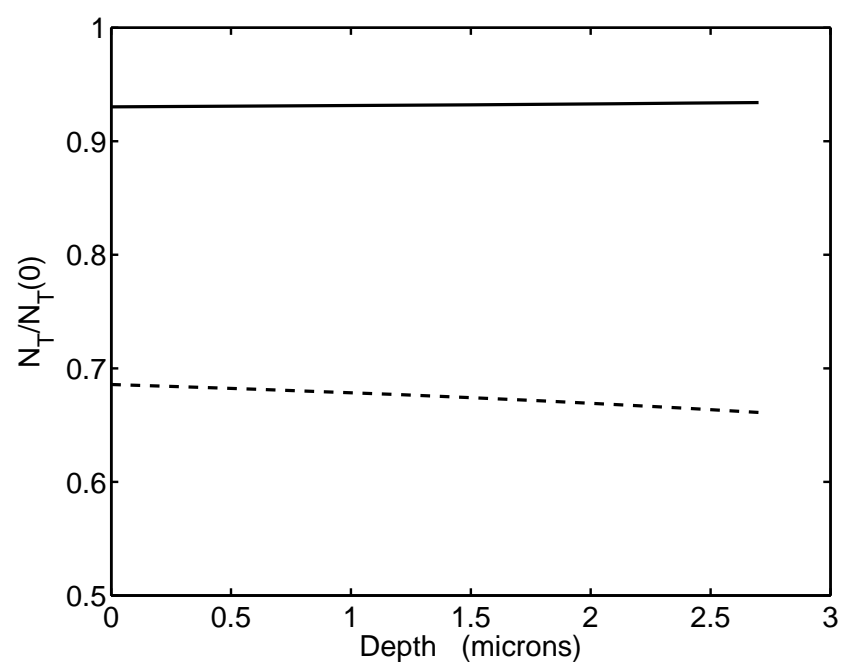

Figure 6: Profile of the ratio of the $E$-center concentration after ICP and before ICP, for 30 minutes ICP on $\mathrm{Au}$ (continuous line) and for the same time of ICP directly on Ge (dashed line). The data has been obtained from the linear fit shown in Figure 5. The reduction of defects is almost constant for the measured depth.

about $20 \%$ for a thickness of $25 \mathrm{~nm}$. Perturbations in metals can be focusons which are known to have a range of the order of tens of lattice sites at room temperature or ILMs which appear in MD in metals $[6,7]$. The properties of localized energy transmission through different metals is a subject of future research.

\subsection{The plasma source}

The plasma beam source (COPRA DN-160) accelerates ions towards an extraction grid from which the beam propagates towards the germanium wafer situated at a distance $x=10 \mathrm{~cm}$ from the grid. This source is described in Ref. [26]. The ion energy distribution function (IEDF) consists of two peaks, a high energy peak (hep) due to the ions in the beam attenuated through charge exchange collisions with the neutrals, and a low energy peak (lep) due to thermalized ions. The high energy peak diminishes rapidly with pressure, according to $I_{\text {hep }}=I_{\text {lep }} \exp \left(-x / l_{\text {ex }}\right)$, being $l_{\text {ex }}$ the mean free path due to the charge exchange collisions and $x$ the distance to the source. At the present experiment's values $p=0.1 \mathrm{mb}, x=10 \mathrm{~cm}$ and $l_{x} \simeq 1 \mathrm{~mm}$ it has completely disappeared. Therefore, the ion flux arriving at the Ge wafer is due to the thermalized second peak, i.e., the ions being accelerated in a collisional sheath [27]. Both the ion energy and the current density $J$ diminish with pressure. The ion energy at the working pressure can be obtained from the data provided by the manufacturer (Figure 7). The current density $J$ at our working pressures was not provided by the manufacturer and we have measured it with a Faraday cup. The corresponding ion flux $\Phi_{i}=J / e$ obtained within the range of accuracy (ion energy above $6 \mathrm{eV}$ ) is shown in Figure. 8. It can be seen that $\Phi_{i} \propto \exp (-\alpha p)$, with $\alpha \simeq 91 \mathrm{mb}^{-1}$, which allows us to obtain the flux at $p=0.1 \mathrm{mb}$ as $\Phi_{i}=5.6 \times 10^{10} \mathrm{~cm}^{-2} \mathrm{~s}^{-1}$. Note that due to charge exchange collisions there is also a flux 


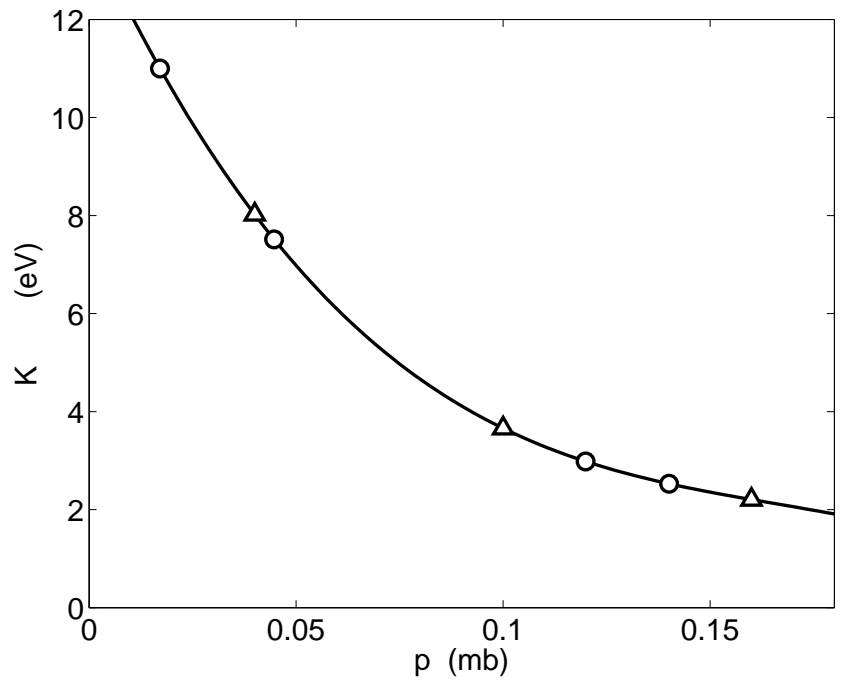

Figure 7: Plasma ion energy with respect to pressure according to the manufacturer (triangles) and the values used in this work (circles).

of energetic neutrals which could increase the bombardment frequency by a factor of the order of the unity.

At the more usual operational pressures of $10^{-3} \mathrm{mb}$, the mean free path for charge exchange is about $1 \mathrm{~cm}$, and most of the beam arrives at the wafer with much larger ion current density.

\subsection{Estimation of the number of ILMs and their effect on annealing}

In the following we try to obtain an estimate of the efficiency of ILM creation by $\mathrm{Ar}^{+}$ hits and subsequent ILM annealing of defects.

As the depth profile of the reduction of defect concentration is almost uniform before and after ICP (Figure 6) we can suppose that a large proportion of ILMs crosses the wafer without interacting with the defects and the flux of ILMs $\Phi_{\text {ILM }}$ is almost uniform over the wafer. The defects are almost isolated due to their low concentration, their mean distance being about $\left(1 / N_{T}\right)^{1 / 3} \simeq 2000 \AA$ or about 400 lattice units. Therefore, we can suppose a first order kinetics

$$
\frac{\dot{N}_{\text {ann,ILM }}}{N_{T}}=-\sigma_{\text {ILM }} \Phi_{\text {ILM }},
$$

where $\sigma_{\text {ILM }}$ is the scattering cross section of ILMs by defects, with $\dot{N}_{T}$ being proportional to $N_{T}$, which is consistent with the experimental data shown in Figure 6 . In the following we will assume a constant annealing rate which is equal to its average during the experiment, as the subsequent calculations are basically accurate within an order of magnitude.

The number of defects annealed can be obtained easily. There are initially $N_{T}=$ $1.07 \times 10^{14} \mathrm{~cm}^{-3}$ defects and $30 \%$ of these are annealed in $t=30$ minutes of ICP up to 


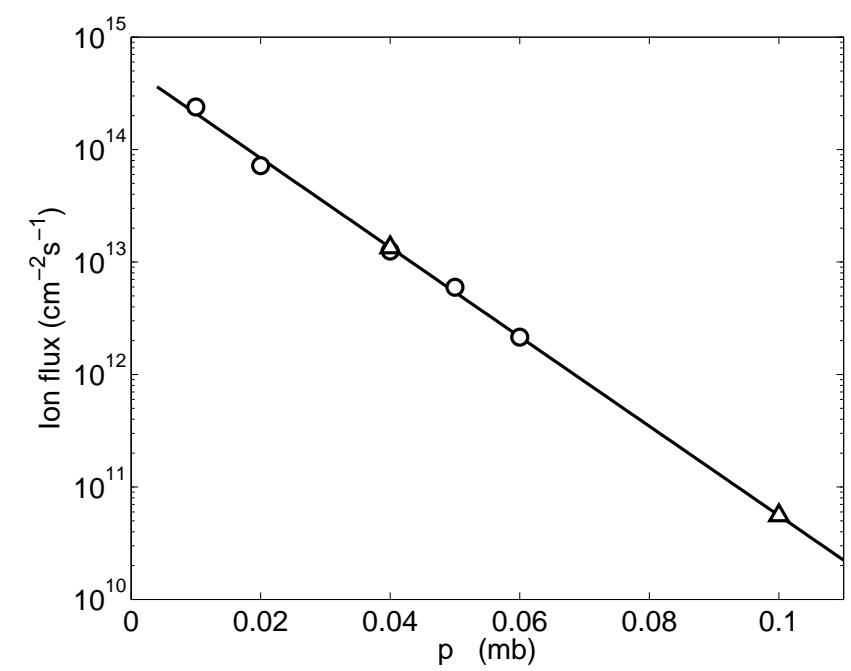

Figure 8: Ion flux $\Phi=J / e$ obtained with a Faraday cup (circles) and the fitted ion flux for $8 \mathrm{eV}(0.4$ $\mathrm{mb})$ and $4 \mathrm{eV}(0.1 \mathrm{mb})$ plasma (triangles)- see text.

a depth exceeding $d=2600 \mathrm{~nm}$. Therefore, the average annealing rate per unit area is given by

$$
\dot{N}_{\text {ann,sup }}=\frac{0.30 N_{T} d}{t} \simeq 4.6 \times 10^{6} \mathrm{~cm}^{-2} \mathrm{~s}^{-1} .
$$

Comparing with the flux of ions calculated above, we can see that approximately $10^{4}$ $\mathrm{Ar}^{+}$hits are needed to anneal a defect.

Let us introduce the parameter $\gamma$, the efficiency of ILM creation, defined by:

$$
\Phi_{\mathrm{ILM}}=\gamma \Phi_{i} .
$$

In principle, we can suppose that $\gamma \leq 1$, as it seems unlikely that an Ar hit will produce more than one ILM.

Let us write $\sigma_{\text {ILM }}=\alpha \sigma_{0}$ with $\alpha \geq 1$ and $\sigma_{0}$ equal to the area occupied at the surface by a Ge atom $\sigma_{0}=\left(n_{\mathrm{Ge}}\right)^{-2 / 3} \simeq 8 \times 10^{-16} \mathrm{~cm}^{2}$. The parameter $\alpha$ is just the cross section of the interaction between ILMs and defects measured in $\sigma_{0}$ units. Most likely it will depend on temperature but here we consider only its value at the experimental temperature.

Therefore

$$
\frac{\dot{N}_{\text {ann,ILM }}}{N_{T}}=-\alpha \gamma \sigma_{0} \Phi_{i} .
$$

Note that the ion-defect cross-section $\sigma_{i}$ defined in Eq. (2) is precisely $\sigma_{i}=\alpha \gamma \sigma_{0}$. The value of the product $\alpha \gamma$ is approximately 3.6. We do not know the values of either $\alpha$ or $\gamma$ but to have an estimation of the numbers involved, the cross section should be larger than $\sigma_{0}$ because on the one hand the size of an $E$-center is at least two atoms, and on the other hand, the ILM will also have some extension perpendicular to the direction 
of its movement. Let us suppose that the interaction takes place for a distance of four atoms, in this case $\sigma_{\mathrm{ILM}}=8^{2} \sigma_{0}$ or $\alpha \simeq 64$, then $\gamma \simeq 0.06$, meaning that about $20 \mathrm{Ar}^{+}$ hits produce an ILM.

We can try different combinations of numbers, whose exact value we do not know at this stage, but what is clear is the extremely high efficiency of these three processes, ILM creation by Ar ion hits, ILM transmission through germanium, and the annealing of defects by ILMs.

\subsection{Comparison between room temperature thermal annealing and ILM annealing}

It is interesting to see that the annealing effect is not simply produced by more energy delivered to the defects. For that we can compare the density of energy for ILMs and for phonons at the temperature where the latter have a similar rate of annealing, which is $T_{\text {ann }}=423 \mathrm{~K}[13,15]$.

If we estimate the speed of ILMs as $v_{\mathrm{ILM}} \simeq 5400 \mathrm{~m} / \mathrm{s}$, the sound velocity in germanium, their energy density is given by:

$$
\rho_{\mathrm{ILM}}=n_{\mathrm{ILM}} E_{\mathrm{ILM}}=\frac{\Phi_{\mathrm{ILM}} E_{\mathrm{ILM}}}{v_{\mathrm{ILM}}},
$$

with $E_{\mathrm{ILM}} \simeq 3 \mathrm{eV}$.

For phonons, we construct a simple model. The density of states of phonons in Ge shows a strong peak of overlapping optical bands between 8 and $9 \mathrm{THz}$ or 33 and 37 $\mathrm{meV}$ at the top of the phonon band [28]. So, let us use an Einstein model of phonons with the same energy $E_{\mathrm{ph}}=35 \mathrm{meV}$. The energy density of phonons at the temperature of annealing $T_{\text {ann }}=423 \mathrm{~K}$ is given by:

$$
\rho_{\mathrm{ph}, \text { ann }}=\frac{3 n_{\mathrm{Ge}} E_{\mathrm{ph}}}{\exp \left(E_{\mathrm{ph}} / k_{B} T_{\mathrm{ann}}\right)-1} .
$$

Supposing $\gamma=1$, the ratio between these two energy densities that brings about the same rate of annealing is $\rho_{\mathrm{ILM}} / \rho_{\mathrm{ph}, \text { ann }} \simeq 10^{-16}$, which shows very clearly that the effect produced by ILMs is not a thermal one.

The other point of view is that low energy, low flux plasmas seem an ideal source to produce ILMs in materials. The energy of each individual ion is of the order of magnitude of what is necessary to produce an ILM, the direction perpendicular to the surface is appropriate for an efficient energy transfer of momentum towards the interior of the semiconductor. The low density and flux of the plasma means that each ion impact is almost isolated. In $0.5 \mathrm{~ns}$ an ILM traverses the $2600 \mathrm{~nm}$ tested depth of the germanium wafer. During that time, no other Ar hit is produced in a circle with a radius $10^{6}$ lattice units, thus there is no possibility of a wavefront being produced or of an ILM being perturbed by another ILM. Of course, a technique as precise and sensitive as DLTS is necessary to appreciate the effect. We should also note that ILMs can not only anneal defects but they can also create or transform them as described in the experiment reported here but also in Ref. [16], where a new defect, known as $\mathrm{E}_{0.31}$ was created deep into Ge by 8-60 eV ICP Ar plasma. 


\section{Conclusion}

In conclusion, it was observed that the interaction of a low energy (4 eV) Ar plasma with the surface of Sb-doped germanium brings about the annealing of defects up to a depth exceeding $2600 \mathrm{~nm}$. Due to the low energy and low flux conditions of the experiment the conclusion is that localized energy of the order of an $\mathrm{eV}$ is able to travel through the crystal. An increase on ion energy does not increase the annealing efficiency but observations to the contrary suggest that most of the excess energy has the effect of hindering the localized energy transmission. Equally, if the semiconductor is allowed to heat up the annealing efficiency decreases. This suggests that the transmission of localized energy in the form of moving intrinsic localized modes takes place. If this hypothesis is correct, ILMs have an amazing capability of annealing defects and of travelling long distances in Ge. On the other hand, low energy plasmas seem an ideal source to produce nonlinear vibrational excitations in crystal, due to their low density, direction of ion impact and energy of the ions similar to that expected for ILMs. Our experiment opens an extended area of experimentation involving nonlinear excitations in semiconductors and metals.

\section{Acknowledgments}

The authors were funded by MICINN, project FIS2008-04848; the South African National Research Foundation and the European Regional Development Fund, Centre of Excellence Mesosystems: Theory and Applications. JFRA and VD acknowledges the Physics Institute in Tartu for their hospitality.

[1] A. J. Sievers, S. Takeno, Intrinsic localized modes in anharmonic crystals, Phys. Rev. Lett. 61 (1988) 970-973.

[2] S. Flach, A. V. Gorbach, Discrete breathers advances in theory and applications, Phys. Rep. 467 (2008) 1-117.

[3] F. M. Russell, J. C. Eilbeck, Evidence for moving breathers in a layered crystal insulator at 300K, Europhys. Lett. 78 (2007) 10004.

[4] J. F. R. Archilla, J. Cuevas, M. D. Alba, M. Naranjo, J. M. Trillo, Discrete breathers for understanding reconstructive mineral processes at low temperatures, J. Phys. Chem. B 110 (47) (2006) $24112-24120$

[5] V. I. Dubinko, A. V. Dubinko, Modification of reaction rates under irradiation of crystalline solids: contribution from intrinsic localized modes, Nucl. Instrum. Meth. B 303 (2013) 133-135.

[6] M. Hass, V. Hizhnyakov, A. Shelkan, M. Klopov, A. J. Sievers, Prediction of high-frequency intrinsic localised modes in Ni and Nb, Phys. Rev. B 84 (2011) 144303.

[7] V. Hizhnyakov, M. Haas, A. Shelkan, M. Klopov, Theory and md simulations of intrinsic localized modes and defect formation in solids, Phys. Scr. 89 (4) (2014) 044003.

[8] C. A. Becker, F. Tavazza, Z. T. Trautt, R. A. Buarque de Macedo, Considerations for choosing and using force fields and interatomic potentials in materials science and engineering, Curr. Opin. Solid State Mater. Sci. 17 (2013) 277-283.

URL http://www.ctcms.nist.gov/potentials

[9] D. V. Lang, Deep-level transient spectroscopy: A new method to characterize traps in semiconductors, J. Appl. Phys. 45 (7) (1974) 3023-3032.

[10] D. K. Schroder, Semiconductor Material and Device Characterization, 3rd Edition, John wiley, New Jersey, USA, 2006, p 260.

[11] F. D. Auret, S. Coelho, G. Myburg, P. J. J. van Rensburg, W. E. Meyer, Defect introduction in Ge during inductively coupled plasma etching and Schottky barrier diode fabrication processes, Thin Solid Films 518 (2010) 2485.

[12] F. D. Auret, P. J. J. van Rensburg, M. Hayes, J. M. Nel, S. Coelho, W. E. Meyer, S. Decoster, V. Matias, A. Vantomme, D. Smeets, Electrical characterization of defects in heavy-ion implanted n-type Ge, Nucl. Instrum. Meth. B 257 (2007) 169. 
[13] V. P. Markevich, A. R. Peakera, V. V. Litvinov, V. V. Emtsev, L. I. Murin, Electronic properties of antimony-vacancy complex in Ge crystals, J. Appl. Phys. 95 (2004) 4078.

[14] J. Fage-Pedersen, A. N. Larsen, Irradiation-induced defects in Ge studied by transient spectroscopies, Phys. Rev. B 62 (2000) 10116.

[15] C. Nyamhere, Characterization of process and radiation induced defects in Si and Ge using conventional deep level transient spectroscopy (DLTS) and laplace-DLTS, Ph.D. thesis, University of Pretoria, viewed 14-03-2014 (2009). URL http://upetd.up.ac.za/thesis/available/etd-02022010-134937/

[16] S. M. M. Coelho, F. D. Auret, P. J. Janse van Rensburg, Unexpected properties of the inductively coupled plasma induced defect in germanium, Physica B 439 (2014) 98-100.

[17] V. Kolkovsky, M. C. Petersen, A. N. Larsen, Alpha-particle irradiation-induced defects in n-type germanium, Appl. Phys. Lett. 90 (11) (2007) 112110

[18] K. Roro, P. Janse van Rensburg, F. Auret, S. Coelho, Effect of alpha-particle irradiation on the electrical properties of n-type Ge, Physica B 404 (22) (2009) 4496-4498.

[19] S. T. Pantelides, A. Oshiyama, R. Car, P. J. Kelly, Theory of electronically stimulated defect migration in semiconductors, Phys. Rev. B 30 (1984) 2260-2262.

[20] J. R. Weber, A. Janotti, P. Rinke, C. G. Van de Walle, Dangling-bond defects and hydrogen passivation in germanium, Appl. Phys. Lett. 91 (14) (2007) 142101.

[21] J. R. Weber, A. Janotti, C. G. Van de Walle, Dangling bonds and vacancies in germanium, Phys. Rev. B 87 (2013) 035203.

[22] S. M. M. Coelho, F. D. Auret, P. J. Janse van Rensburg, J. Nel, Electrical characterization of defects introduced in n-Ge during electron beam deposition or exposure, J. Appl. Phys. 114 (17) (2013) 173708.

[23] H. Tahini, A. Chroneos, R. W. Grimes, Schwingenschlo, H. Bracht, Diffusion of E centers in germanium predicted using GGA+U approach, Appl. Phys. Lett. 99.

[24] V. Hizhnyakov, D. Nevedrov, A. J. Sievers, Quantum properties of intrinsic localized modes, Physica B 316-317 (2002) 132-135.

[25] N. K. Voulgarakis, G. Hadjisavvas, P. C. Kelires, G. P. Tsironis, Computational investigation of intrinsic localization in crystalline Si, Phys. Rev. B 69 (2004) 1132001.

[26] R. Hippler, J. Kredl, V. Vartolomei, Ion energy distribution of an inductively coupled radiofrequency discharge in argon and oxygen, Vacuum (2009) 732-737.

[27] J. Liu, G. Huppert, H. Sawin, Ion bombardment in rf plasmas, J. Appl. Phys. 68 (8) (1990) 39163934.

[28] S. Wei, M. Y. Chou, Phonon dispersion of silicon and germanium from first principles calculations, Phys. Rev. B 50 (1994) 2221. 